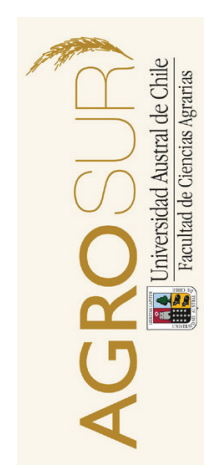

\title{
Incidencia de variables biológicas y edáficas en el establecimiento de mezclas forrajeras
}

\author{
Incidence of biological and edaphic variables \\ on the establishment of forage mixtures
}

\author{
García-Favre, J. ${ }^{a *}, Z^{2}$ anoniani, R. ${ }^{a}$, Cadenazzi, M. ${ }^{b}$, Boggiano, P. ${ }^{a}$ \\ ${ }^{a}$ Departamento de Producción Animal y Pasturas, Facultad de Agronomía, UDELAR. \\ ${ }^{b}$ Departamento de Biometría, Estadística y Computación, Facultad de Agronomía, UDELAR.
}

\begin{abstract}
ARTICLE INFO
Article history:

Received 04.04.2017

Accepted 23.05.2017

Keywords:

Decision tree

Species competition

Predecessor crop

Original Research Article,

Animal Science

*Corresponding author:

Javier García-Favre

E-mail address:

javigarciafavre@gmail.com
\end{abstract}

A B S T R A C T

With the aim of evaluating the incidence of biological and edaphic factors on the establishment of forage mixtures, a trial was set consisting in 180 sites in the states of Florida, Durazno and Lavalleja, Uruguay. The percentage of establishment was measured 90 days after seeding through the percentage of emergency, in three types of mixtures: a) perennial grass and legumes; b) perennial grass and legumes, and annual ryegrass; c) perennial grass and legumes, and wheat. Biological (predecessor crop, predecessor crop stubble, date of seeding, species sown) and edaphic (organic matter, $\mathrm{pH}$, phosphorus) factors were quantified to determine their effects on the establishment period of the sowed seeds. This period was $29.2 \%$ for all the sites and species. Type of predecessor crop and species sowed (Festuca arundinacea Schreb., Dactylis glomerata L., Trifolium repens L., Trifolium pratense L., Lotus corniculatus L., Medicago sativa L., Lolium multiflorum Lam., Triticum aestivum L.) in the mixtures were determinant in the establishment percentage of the mixtures (perennial grass and legumes). Annual grasses reached the highest establishment percentage (50\%) but supressed perennial grasses. Setaria italica (L.) P. Beauv., was the best predecessor crop allowing an establishment of $40 \%$ in a mixture of perennial grass and legumes. On the other hand, Lotus corniculatus achieved the lowest establishment (14\%). A negative correlation was found between legume establishment and a pH lower than 5.9.

\begin{abstract}
RESUMEN
Con el objetivo de evaluar la incidencia de factores biológicos y edáficos en el establecimiento de mezclas forrajeras, se efectuó un experimento que abarcó 180 sitios en los departamentos de Florida, Durazno y Lavalleja, Uruguay. El porcentaje establecimiento fue medido a los 90 días de la siembra en tres tipos de mezclas: a) gramíneas y leguminosas perennes; b) gramíneas y leguminosas perennes, y raigrás anual; c) gramíneas y leguminosas perennes, y trigo. Fueron cuantificados distintos factores biológicos (tipo de rastrojo, fecha de siembra, especies sembradas) y edáficos (materia orgánica, $\mathrm{pH}$ y fósforo), para determinar las causas que afectaron el establecimiento de las semillas sembradas. El establecimiento promedio para todos los sitios y especies fue de 29,2\%. El cultivo antecesor y el tipo especie sembrada en la mezcla (Festuca arundinacea Schreb., Dactylis glomerata L., Trifolium repens L., Trifolium pratense L, Lotus corniculatus L., Medicago sativa L., Lolium multiflorum Lam., Triticum aestivum L.) fueron determinantes en el porcentaje de establecimiento de las mezclas. Las gramíneas anuales fueron las de mayor establecimiento (50\%) pero deprimieron a las especies perennes. Setaria italica (L.) P. Beauv., fue el antecesor más apto (40\%) para el establecimiento de las gramíneas perennes y leguminosas. Por el contrario, Lotus corniculatus alcanzó el menor establecimiento (14\%). Asimismo, se encontró una correlación negativa entre el establecimiento de leguminosas y nivel de pH menores a 5,9.
\end{abstract}

Palabras clave: árbol de decisión, competencia de especies, cultivo antecesor.

\section{INTRODUCCIÓN}

Las praderas cultivadas por su alta calidad y producción son una alternativa utilizada con frecuencia en Uruguay, como complemento a la pradera natural. Sin embargo, el área sembrada de praderas cultivadas ha disminuido en la última década, de 1,4 millones de hectáreas en 2006, a 1,01 millones de hectáreas en 2015 (MGAP/DIEA, 2015). Esta disminución es de- bida, entre otras razones, a su escasa persistencia, lo cual ha favorecido su sustitución por la siembra de mayor superficie de pasturas anuales de invierno. El enmalezamiento temprano, las fechas de siembras tardías, la inestabilidad de las mezclas, el cultivo antecesor, el inadecuado establecimiento y el mal manejo de las praderas son las causas de su baja producción y persistencia, desincentivando la siembra de praderas (Zanoniani, 2010). 
El establecimiento de las especies sembradas requiere disponer de los insumos tecnológicos adecuados y los conocimientos necesarios para llevarla a cabo. Generalmente, las semillas forrajeras son de tamaño pequeño, presentan escasas reservas, tienen un crecimiento inicial lento, poca habilidad competitiva frente a malezas y son susceptibles a enfermedades y plagas (Carámbula, 1991). El éxito de la siembra en cuanto al número de plantas establecidas depende de que se brinden las condiciones necesarias para romper la dormancia y que se aseguren los procesos de germinación y protección contra depredadores (Harper y Benton, 1966).

En las praderas cultivadas la proporción de plantas establecidas respecto de las semillas viables sembradas se determina en las primeras semanas luego de la siembra. En este período la competencia es intensa entre las plántulas por luz, agua y nutrientes. Sólo las especies más vigorosas y con capacidad para soportar ataques de insectos y enfermedades y condiciones desfavorables de temperatura y humedad van a lograr establecerse de manera adecuada (Formoso, 2010).

Una característica importante de las semillas es su capacidad de movilizar reservas de carbohidratos desde el endosperma hacia los puntos de crecimiento, existiendo diferencias entre especies en la rapidez de movilización de reservas (Askin, 1994) que determinan diferencias en la velocidad de germinación; el raigrás anual es un claro ejemplo de esto, presenta mayor tasa de desdoblamiento del endosperma, comparativamente a otras gramíneas (Brock et al., 1982).

Por otro lado, diferencias morfológicas y fisiológicas de los sistemas radicales influyen en la eficiencia con la cual las especies pueden obtener y competir por nutrientes y agua. Las raíces de forma fasciculada de las gramíneas son más densas por unidad de volumen de suelo y, en consecuencia, poseen una mayor superficie de contacto con el suelo, cuando se las compara con las raíces pivotantes de las leguminosas. Evans citado por Harris (1994) consideró que esto podría darles a las gramíneas una ventaja competitiva sobre las leguminosas, en la obtención de nutrientes y agua, y en especial de nutrientes poco móviles como fósforo.

Hay factores extrínsecos a las características de la planta que afectan los procesos mencionados. El cultivo antecesor determina o condiciona el tipo de rastrojo presente en el suelo, que conlleva a una mayor o menor inmovilización de nutrientes dependiendo de su relación de C/N (Loydi et al., 2013). También relacionado al cultivo antecesor y al barbecho, la presencia de restos vegetales en superficie afecta los niveles de humedad y temperatura del suelo, y determinan la calidad y cantidad de luz que llega a las semillas y plántulas. En efecto, la intercepción por estos restos reduce la cantidad de luz que llega al suelo, y modifican la relación rojo/rojo lejano, que regula el proceso de germinación, pudiendo ser inhibida en las semillas sembradas (Deregibus et al., 1994).

Se debe tener en cuenta, que la descomposición de los rastrojos es un proceso complejo y prolongado cuya tasa varía con la naturaleza del sustrato y las condiciones del medio (Satchell, 1974). Asimismo, su calidad influye en la velocidad y en el tipo de descomposición, ya sea por bacterias encargadas de descomponer aquellos compuestos más fáciles o por hongos en los casos de los compuestos de degradación más difícil: lignina, celulosa y hemicelulosa (Borges, 2001). La relación $\mathrm{C} / \mathrm{N}$ es uno de los parámetros utilizados para caracterizar la calidad de los rastrojos. La tasa de descomposición está afectada por la cantidad de sustancias solubles en agua, sustancias lixiviables, contenido de $\mathrm{N}$ y polifenoles de restos frescos, siendo esta mayor cuando la relación $\mathrm{C} / \mathrm{N}$ es más baja. Además, los rastrojos en superficie tienden a favorecer relativamente el desarrollo de hongos frente a las bacterias, estos microorganismos tienen una mayor relación C/N en sus tejidos pero son más eficientes en la conversión del carbono del substrato a sus tejidos determinando mayores niveles de inmovilización de $\mathrm{N}$ en siembra directa (Morón, 2001).

La competencia de las malezas afecta el establecimiento y la dominancia de una especie en la pradera. Donald (1963) la define como un fenómeno que ocurre cuando dos o más organismos o poblaciones requieren una cierta cantidad de un factor particular, en condiciones en que la capacidad del ambiente de suministrarlo en forma inmediata es inferior a la demanda combinada de los organismos involucrados; siendo los ambientes de los organismos vecinos interdependientes. En este sentido, De Wit (1960), utilizó para medir la competencia en el periodo de establecimiento de praderas, la cuantificación de la sobrevivencia de cada especie en relación al número de semillas viables sembradas.

El objetivo del presente trabajo fue estudiar la forma y la magnitud en que se afectó el establecimiento de pasturas cultivadas al variar el cultivo antecesor, las especies sembradas, la fecha de siembra y las características químicas del suelo.

\section{MATERIALES Y MÉTODOS}

Entre marzo y octubre del 2007 se realizó el relevamiento de un total de 180 sitios, distribuidos en los departamentos de Florida, Durazno y Lavalleja. Dichos sitios corresponden a grupos de suelos CONEAT $10.3 \mathrm{y}$ 5.02b, predominando suelos Brunosoles Eútricos Lúvicos y/o Háplicos, asociados a Inceptisoles en los suelos 5.02b (CONEAT, 1979).

La falta de información previa sobre los sitios en estudio llevó a realizar un muestreo aleatorio irrestricto, donde la unidad de estudio fue la pradera y la unidad de muestreo cada uno de los 180 sitios. 
La variable de repuesta estudiada fue el porcentaje de establecimiento a los 90 días de la siembra (IMP). Los factores considerados fueron a) cultivo antecesor: Sorghum sudanense (Piper) Stapf. (sorgo pastoreo)(1), Sorghum sudanense $\mathrm{x}$ bicolor BMR silero (2), Sorghum bicolor Moench (sorgo granífero)(3), Zea mays Linnaeus (maíz)(4), Glycine max Linnaeus (soja)(5), Setaria italica Linnaeus (moha)(6), Avena byzantiva Koch (avena) (7), Lolium multiflorum Lamarck (raigrás anual)(8), Triticum aestivum Linnaeus (trigo)(9), praderas cultivadas de más de cuatro años con alto grado de enmalezamiento por gramíneas perennes de ciclo estival (pradera vieja)(10) y pastizales nativos (campo natural) (11); b) Especie sembrada: Festuca arundinacea Schreber (festuca), Dactylis glomerata Linnaeus (dactylis), Trifolium repens Linnaeus (trébol blanco), Trifolium pratense Linnaeus (trébol rojo), Lotus corniculatus Linnaeus, Lolium multiflorum Lamarck, Triticum aestivum Linneaus; c) Fecha siembra: Abril, Mayo, Junio y Julio; d) Composición de la mezcla de la pradera: gramínea perenne (festuca o dactylis)-leguminosa (1), gramínea perenne (festuca o dactylis)-leguminosa-raigrás anual (2), gramínea perenne (festuca o dactylis)-leguminosatrigo (3); e) Nivel de fósforo (P); f) Nivel de materia orgánica (MO); g) Nivel de acidez del suelo $(\mathrm{pH})$.

Estos tres últimos atributos químicos del suelo fueron medidos al momento de la siembra de las praderas a $20 \mathrm{~cm}$ de profundidad. Para la determinación fósforo se utilizó el método de Bray y Kurtz (1945), para materia orgánica el método usado fue el de Walkley y Black (1934), mientras que, el nivel de pH se determinó mediante un potenciómetro.

Las determinaciones se realizaron en 2 momentos. La primera fue realizada a la siembra, donde se describían los factores a considerar (fecha siembra, análisis de suelo, antecesor, semilla utilizada, y aspectos de la siembra en sí). A su vez se tomaron muestras de semilla para cuantificar, en laboratorio, pureza, peso de mil semillas y germinación.

A los 90 días después de la siembra se midió el número de plantas $\mathrm{m}^{-1}$ lineal (especies sembradas en la línea) y con cuadros (especies sembradas al voleo), para obtener el número de plantas $\mathrm{m}^{-2}$ logradas. Utilizando la información de kg sembrados y, las características obtenidas del análisis de las semillas (peso mil semillas, \% de germinación y pureza), se calculó el número de semillas viables sembradas. Con esta información y el número de plantas $\mathrm{m}^{-2}$ obtenidas se procedió a calcular el porcentaje de establecimiento.

Las mezclas utilizadas estaban constituidas con $8 \mathrm{~kg} \mathrm{ha}^{-1}$ de Lotus corniculatus, $2 \mathrm{~kg} \mathrm{ha}^{-1}$ trébol blanco, o $6 \mathrm{~kg} \mathrm{ha}^{-1}$ de trébol rojo y $15 \mathrm{~kg} \mathrm{ha}^{-1}$ de festuca o $10 \mathrm{~kg}$ ha $^{-1}$ de dactylis (1). La constitución de la mezcla (2) incorporó a la anterior mezcla $15 \mathrm{~kg} \mathrm{ha}^{-1}$ de raigrás anual. Mientras que, la mezcla (3) se constituyó por la (1) más $60 \mathrm{~kg} \mathrm{ha}^{-1}$ de trigo.
Se realizaron análisis multivariados con el programa estadístico R (versión 3.3.0), los que incluyeron análisis de conglomerado con método Ward y distancia Gower (Everitt y Hothorn, 2011a), análisis de componentes principales (Everitt y Hothorn, 2011b), y árboles de clasificación y regresión (Flores et al., 2016). Los análisis de conglomerados y de árboles de clasificación y regresión fueron realizados con las 180 unidades de muestreo (sitios) con todos los factores medidos. El análisis de componentes principales se efectuó en la misma cantidad de sitios pero sólo con los factores de suelo (P, pH, MO e IMP) con el objetivo de buscar asociaciones entre ellos.

\section{RESULTADOS Y DISCUSIÓN}

\section{Efecto de las variables en el establecimiento}

Mediante un análisis de conglomerados se discriminaron 6 grupos o "ambientes", para los 180 sitios muestreados. Para determinar el número de grupos se utilizó la suma de cuadrados del error dentro de los mismos, la cual a partir de 6 no presenta una disminución acentuada (Figura 1).

El promedio de establecimiento para los 180 sitios de muestreo fue de 29,2\%. Boschi et al. (2016) citando a varios autores muestran porcentajes de establecimiento de 10 a 59\%, entre los 20 y 50 días después de la siembra en condiciones experimentales. Los resultados de porcentajes de establecimiento a los 90 días después de la siembra, mostraron una alta variabilidad (Cuadro 1). Los grupos 3 y 4 fueron los de mayor establecimiento con 36,5 y $30,7 \%$ respectivamente, mientras que el grupo 1 fue el de menor porcentaje de establecimiento $(21,7 \%)$. Los "ambientes" con mayor establecimiento son los que poseen los promedios más altos en valores relativos de $\mathrm{MO}$ y $\mathrm{pH}$. El P no muestra

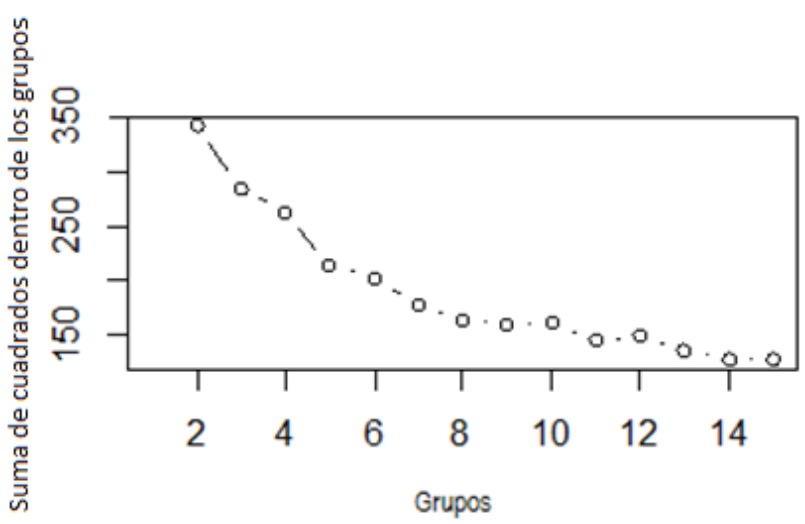

Figura 1. Suma de cuadrados dentro de los grupos de los grupos.

Figure 1. Sum of squares within groups. 
la misma tendencia, ya que el ambiente 3 posee el menor valor. Además, estos ambientes $(3,4$ y 5 ) tienen el antecesor sorgo granífero con mayor frecuencia dentro de estos grupos (Cuadro 1).

Por otro lado, las diferencias en el establecimiento entre grupos no pueden ser explicadas por la variación promedio de los factores (Cuadro 2). Por esta razón, se decidió realizar un análisis de árboles de clasificación y regresión (CART).

\section{Efecto de la especie y cultivo antecesor}

En el árbol de regresión utilizando como variable respuesta establecimiento (IMP) se puede apreciar que la especie es la primera variable clasificatoria $(\mathrm{P}<0,001)$ (Figura 2). Las gramíneas anuales, trigo y raigrás anual, son las especies que mejor establecimiento presentaron (50\%). Esto en trigo, es atribuible al tamaño de las semillas, el cual está relacionado con el peso de la misma. En general, a mayor peso, mayor tamaño, la velocidad de germinación es superior por aumento del contenido de endosperma en la semilla; lo que incrementa el vigor de plántulas, los porcentajes de establecimento, la producción de raíces y la sobrevivencia bajo ambientes estresantes, reduciendo el tiempo para iniciar el crecimiento de tallos (Formoso, 2007). En raigrás anual, que posee menor tamaño de semillas en comparación con otras gramíneas, su mayor establecimiento comparativamente con gramíneas perennes, tales como festuca, se pudo deber a la mayor tasa de crecimiento relativa (Garnier, 1992) que resulta en plantas más desarrollas que compiten mejor por recursos limitantes en un período de tiempo dado. Además ésta especie, presenta mayor tasa de movilización de reservas de almidón del endosperma hacia el embrión, que especies perennes como la festuca (Brock et al., 1982), posibilitando el logro de un mayor vigor de plántulas.
La segunda variable clasificatoria fue el cultivo antecesor $(\mathrm{P}<0,001)$ que tiene incidencia sobre las gramíneas perennes y leguminosas. El sorgo granífero, moha y soja son los antecesores que presentaron mejor establecimiento (40\%) para las especies mencionadas.

Este comportamiento coincidió con lo afirmado por Patrick (1971), que los factores principales en determinar los efectos del rastrojo en el crecimiento y rendimiento del cultivo siguiente, son tipo de rastrojo, cantidad y distribución de las lluvias y grado de descomposición que tenía el rastrojo antes de sembrar el siguiente cultivo. En este sentido, Loydi et al. (2013) a partir del estudio de 46 trabajos independientes, llegaron a la conclusión que los residuos presentes en el suelo tienen un efecto negativo en la germinación de las semillas y en el establecimiento de las especies en diferentes ecosistemas. Además, constataron que la cantidad de biomasa de residuo a partir de la cual el efecto es marcadamente negativo en la germinación y sobrevivencia de las plántulas es de $500 \mathrm{~g} \mathrm{~m}^{-2}$. Resultados similares fueron hallados por Miglécz et al. (2013) que encontraron que las semillas pequeñas fueron

Cuadro 2. Media, desvío estándar (SD), repeticiones (n) de los factores cuantitativos. P: fósforo (\%); MO: materia orgánica (\%); IMP: establecimiento (\%).

Table 2. Mean, standard deviation (SD), replicates (n) of quantitative factors. P: phosphorus (ppm); MO: organic matter (\%); IMP: establishment (\%).

\begin{tabular}{ccccc}
\hline Estadística & $\mathrm{P}$ & $\mathrm{MO}$ & $\mathrm{pH}$ & $\mathrm{IMP}$ \\
\hline Media & 13,9 & 4,4 & 5,4 & 29,2 \\
SD & 10,6 & 0,9 & 0,4 & 17,5 \\
$\mathrm{n}$ & 180 & 180 & 180 & 180 \\
\hline
\end{tabular}

Cuadro 1. Valores promedios de las variables que definen los 6 grupos, discriminados a través de análisis de cluster. Imp: establecimiento (\%); P: fósforo (ppm); M0: materia orgánica (\%). Antecesor: 1 (Sorgo pastoreo); 4 (Maíz); 3 (Moha). Mezcla: 1 (gramínea perenne-leguminosa); 2 (gramínea perenne-leguminosa-raigrás); 3 (gramínea perenne-leguminosa-trigo).

Table 1. Average values of the variables that define the 6 groups, discriminated through cluster analysis. Imp: establishment (\%); P: phosphorus (ppm); MO: organic matter. Predecessor crop: 1 (sorghum grazing); 4 (Corn); 3 (Foxtail millet). Mixture: 1 (perennial grasses-legumes); 2 (perennial grasses-legumes-ryegrass); 3 (perennial grasses-legumes-wheat).

\begin{tabular}{cccccccc}
\hline Grupo & Establecimiento (IMP) & $\mathrm{pH}$ & $\mathrm{P}$ & MO & Antecesor & Mezcla & Fecha de siembra \\
\hline 1 & 21,7 & 5,1 & 11,1 & 3,2 & 1 & 2 & abril \\
2 & 23,1 & 5,2 & 13,9 & 4,1 & 4 & 2 & abril \\
3 & 36,5 & 5,3 & 10,1 & 5,0 & 3 & 3 & mayo \\
4 & 30,7 & 5,6 & 17,2 & 4,5 & 3 & 1 & mayo \\
5 & 29,9 & 5,5 & 12,3 & 4,6 & 3 & 1 & abril \\
6 & 28,9 & 5,6 & 17,7 & 4,4 & 6 & 1 & mayo \\
\hline
\end{tabular}




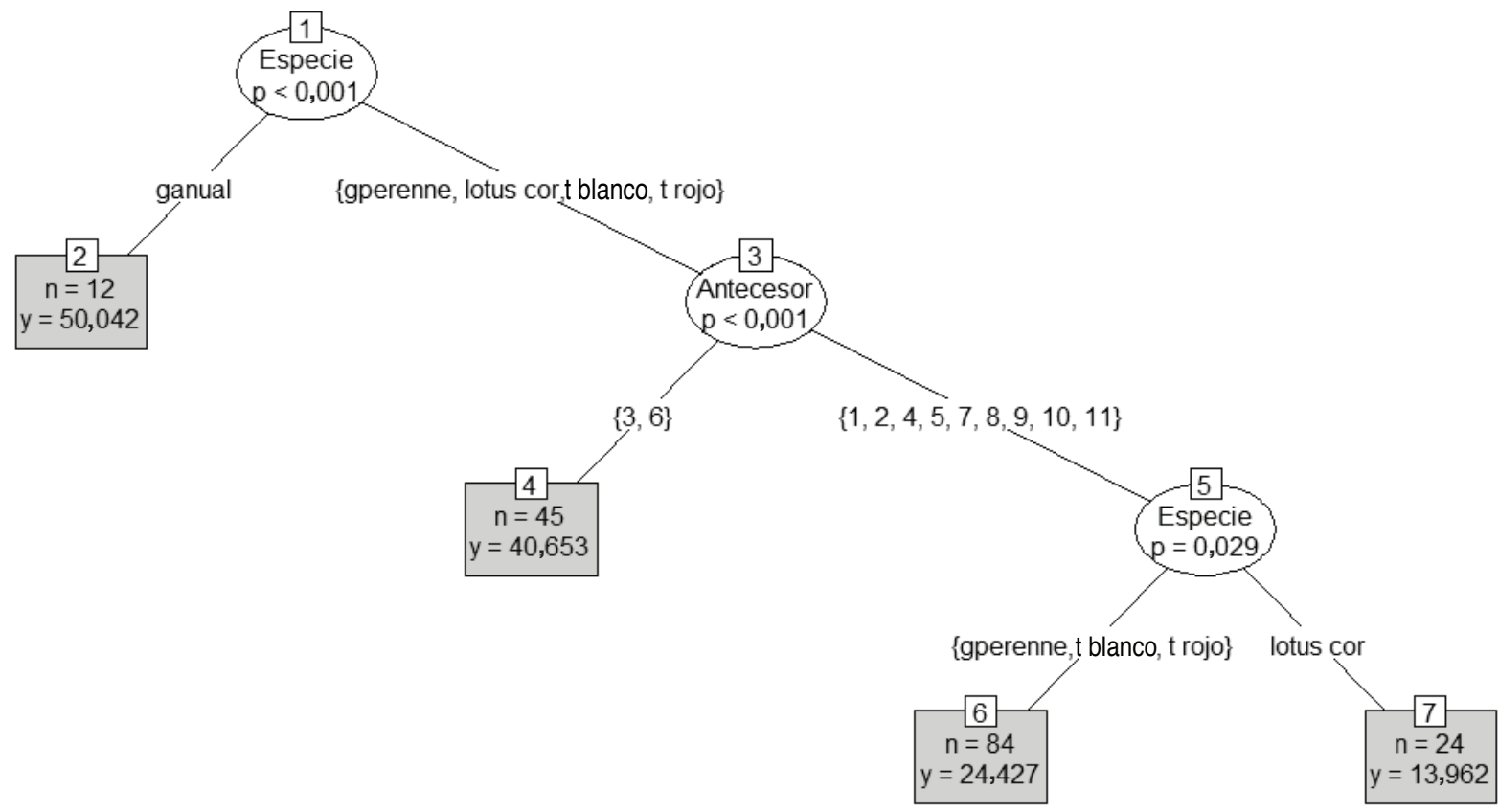

Figura 2. Árbol de regresión para los factores estudiados con porcentaje de establecimiento como variable respuesta. Ganual: gramínea anual (trigo, raigrás anual); gperenne (festuca, dactylis); lotus cor: Lotus corniculatus; t blanco: trébol blanco; t rojo: trébol rojo; 1: sorgo pastoreo; 2: sorgo silero; 3: sorgo granífero; 4: maíz grano; 5: soja, 6: moha; 7: avena; 8: raigrás anual; 9: trigo; 10: pradera vieja; 11: campo natural.

Figure 2. Regression tree for the studied factors with establishment percentage as variable response. Ganual: annual grass (wheat, ryegrass); gperenne (tall fescue, cock's-foot); lotus cor: Lotus corniculatus; t blanco: White clover; t rojo: red clover; 1 : grazing sorghum; 2: whole-plant silage sorghum; 3: grain sorghum; 4: grain maize; 5: soybean, 6: foxtail millet; 7: oat; 8: annual ryegrass; 9: wheat; 10: old pasture; 11: natural pasture.

afectadas negativamente por la presencia de entre 300 y $600 \mathrm{~g} \mathrm{~m}^{-2}$ de rastrojo en superficie. Sin embargo, ambos autores no hallaron efectos negativos para las semillas de gran tamaño; coincidiendo con los resultados que muestra el árbol de regresión (Figura 2), donde las gramíneas anuales presentan el mejor establecimiento sin influencia del antecesor, debiéndose particularmente a la presencia de trigo en éstas.

La moha, como antecesor presentó un alto porcentaje de establecimiento (40\%), se caracteriza por tener una relación $\mathrm{C} / \mathrm{N}$ baja. Posiblemente sea el antecesor más seguro, libera temprano el potrero, controla bien las malezas, provee el suelo con macroporos por su desarrollo radical y deja poca cantidad de rastrojos en superficie (Pozzolo, 2006). Este cultivo presenta ciertas ventajas frente al sorgo, ya que deja un rastrojo de fácil manipulación, apto para la instalación de pasturas anuales de invierno. En este mismo sentido la siembra de este cultivo constituye un buen antecesor para las pasturas perennes, debido a que permite controlar malezas estivales y dado su corto ciclo es posible realizar un buen barbecho previo a la siembra de los mismos (Améndola y Armentano, 2003).

Por lo mencionado anteriormente, podría esperarse que el sorgo granífero con valores de $\mathrm{C} / \mathrm{N}$ de
137 para cañas y 43 para láminas (Morón, 2001) y la presencia de una gran cantidad de rastrojo en superficie luego de la cosecha, no fuera un antecesor propicio como la moha. Sin embargo, la estructuración que provoca en el suelo por la densidad de sus raíces permite una excelente aireación. Sumado a que en el año 2007 las intensas lluvias de marzo (Figura 3) provocaron un atraso en la fecha de siembra y favorecieron la descomposición de parte de los restos vegetales y sobre todo de las raíces, podría explicar la obtención de porcentajes de establecimiento similares a la moha. En condiciones naturales, las tasas de descomposición las regulan temperatura y humedad. A su vez la aireación del suelo, $\mathrm{pH}$ y disponibilidad de nutrientes juegan un papel fundamental debido a que actúan directamente sobre los microorganismos del suelo. La descomposición requiere de agua para el crecimiento microbiano, para la difusión de nutrientes y para la hidrólisis durante los procesos metabólicos (Parr y Papendick, 1978). Asimismo, condiciones ambientales propicias (agua, nutrientes, $\mathrm{pH}$ ) se traducen en la práctica en menores riesgos de perder las pasturas, mayores tasas de crecimiento inicial y mayor precocidad en entregar rendimientos de materia seca superiores (Formoso, 2007). 


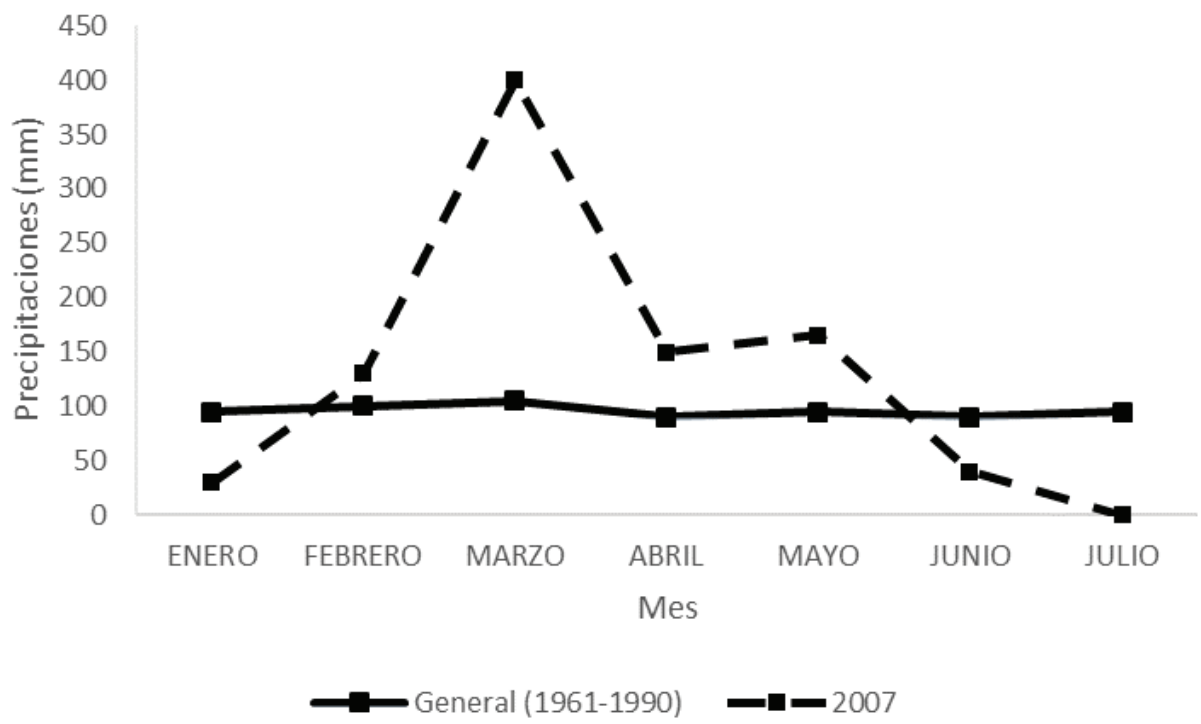

Figura 3. Promedio histórico y del año del relevamiento de precipitaciones mensuales en el centro-sur de Uruguay.

Figure 3. Historical and year of survey monthly rainfall average in south-central Uruguay.

En el extremo opuesto, el sorgo pastoreo como antecesor fue el que peor desempeño tuvo en el establecimiento de las especies con un promedio de $21,7 \%$ (Cuadro 1). Esto se podría atribuir, principalmente a efectos alelopáticos para algunas especies (Milchunas et al., 2011), suelo compactado en superficie por el pastoreo y la presencia en el horizonte superior de una abundante masa radicular difícil de degradar en el corto plazo (Pozzolo, 2006). Además, esta alternativa es muy común que se pastoree hasta entrado el otoño para cubrir las deficiencias otoñales de forraje, lo que limita su capacidad de degradación de la biomasa residual al disminuir las temperaturas (Zanoniani, 2010).

Continuando con análisis de las especies, y siguiendo el gráfico binario de regresión, se encontró que la especie fue nuevamente clasificatoria $(\mathrm{P}=0,029)$. Se observó que por un lado se encontraban las gramíneas perennes, trébol blanco y trébol rojo con un establecimiento promedio de $24,5 \%$. Por el otro, Lotus corniculatus obtuvo el porcentaje de establecimiento más bajo (14\%) (Figura 2). Esto último, denota que el tipo de especie presenta mayor influencia sobre el establecimiento. En este sentido, el género Lotus se ve severamente afectado por enfermedades de establecimiento conocidas como "damping-off". Patógenos del género Pythium spp. y en menor medida Fusarium y Rhizoctonia son los agentes causantes de la muerte de plántulas de la especie, sobre todo en condiciones de excesos hídricos (Pérez et al., 2001). Conviene recordar que el año en el que se realizó el estudio (2007) presentó precipitaciones que estuvieron por encima de las medias mensuales para la época de relevamiento (Figura 3), que pudieron determinar el comportamiento presentado por Lotus corniculatus.

\section{Efecto del pH, MO y P del suelo en el establecimiento}

Las propiedades físicas y químicas del suelo determinan la naturaleza del ambiente donde se encuentran los microorganismos, afectando la población microbiana en forma cuantitativa y cualitativa (Parr y Papendick, 1978). Asimismo, las propiedades químicas del suelo condicionan la sobrevivencia de las plantas ya que crean ambientes más o menos propicios para estas. Este aspecto se puede observar a través del análisis de componentes principales para las características químicas del suelo. En donde se refleja la asociación del $\mathrm{pH}$ con el porcentaje de establecimiento (Figura 4), los 2 componentes principales explican el $64,1 \%$ de la variación.

Sin embargo, el nivel de $\mathrm{pH}$ no posee la misma incidencia en todas las especies. Al analizar el porcentaje de establecimiento a través de árboles de regresión y clasificación, sólo con los factores químicos de suelo (pH, MO, P) para gramíneas por un lado y leguminosas por otro; se encontró que el pH sólo influyó en el porcentaje de establecimiento de las leguminosas $(\mathrm{P}<0,001)$. Mientras que, ningún factor químico del suelo es clasificatorio para las gramíneas.

En la Figura 5 se observa que valores de pH menores a 5,9 determinan que el porcentaje de establecimiento en las leguminosas fuera de $23,3 \%$, mientras que con pH mayor a 5,9 el establecimiento fue más del doble, promediando un 52\% (Figura 5).

En este sentido, George et al. (2012), destacan que en suelos ácidos las mayores restricciones al crecimiento de las plantas son la toxicidad de protones, $\mathrm{Al} \mathrm{y}$ Mn y la deficiencia de Mg, Ca, P y Mo. La importancia re- 
lativa de estas restricciones depende de las especies y genotipos; el tipo y horizonte de suelo; niveles de $\mathrm{pH}$ y tipos de Al; estructura y aireación del suelo; y clima. La movilización de aluminio en la solución del suelo cuando el pH es bajo, puede llegar a alcanzar niveles tóxicos para las plantas. Siendo las leguminosas, en especial los géneros Medicago y Trifolium, más susceptibles a la toxicidad del aluminio, ya que puede tener un efecto perjudicial en la simbiosis Rhizobium-leguminosa, interfiriendo en el proceso de nodulación y por ende el suministro de nitrógeno a la planta (Wright, 1989).

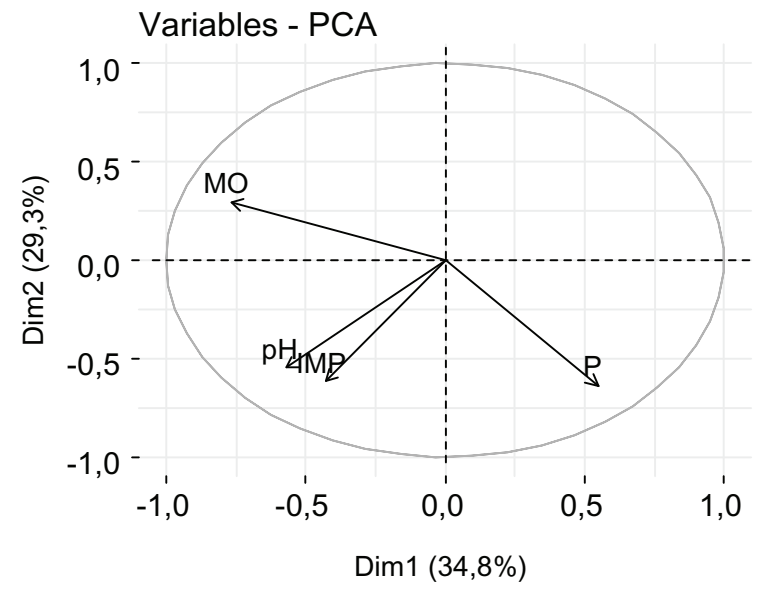

Figura 4. Asociación de los factores fósforo, nivel $\mathrm{pH}$, materia orgánica y la variable establecimiento. IMP: \% establecimiento. P: fósforo. MO: materia orgánica.

Figure 4. Association between phosphorus, pH level, organic matter factors and the variable establishment. IMP: \% of establishment. P: phosphorus. MO: organic matter.

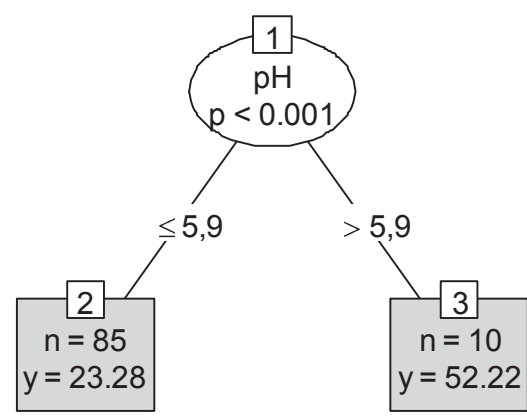

Figura 5. Árbol de regresión para leguminosas. La variable respuesta fue \% de establecimiento y los factores fueron nivel de $\mathrm{pH}$, nivel de fósforo y \% de materia orgánica. 1, 2 y 3 indican la secuencia de decisión del árbol.

Figure 5. Regression tree for legumes. The variable response was $\%$ of establishment and the factors were level of $\mathrm{pH}$, level of phosphorus and \% of organic matter. 1, 2 and 3 show the sequence of decision of the tree.

\section{CONCLUSIONES}

Los resultados de establecimiento obtenidos, tanto el promedio general como el de gramíneas perennes, son bajos al compararlos con cultivos agrícolas. Ambos resultados están cercanos al $30 \%$ a los 90 días del primer año.

El menor porcentaje de establecimiento se obtuvo cuando la combinación de cultivo antecesor y especie fue la siguiente: antecesores campo natural, pradera vieja, maíz y sorgo pastoreo, con mucho rastrojo en superficie y de difícil descomposición (maíz) o muy enmalezados con predominio de gramíneas C4; y la especie Lotus corniculatus.

En cuanto al uso de gramíneas perennes, se concluye que, para obtener un buen establecimiento y así lograr una adecuada población de plantas, es recomendable la no inclusión en la mezcla de gramíneas anuales, las cuales bajan notoriamente el establecimiento de la perenne por su gran vigor y competencia.

Otro aspecto importante es el uso de antecesores como Moha o Soja. Si se elige como cultivo antecesor sorgo granífero, se debe tener en consideración una siembra lo más temprana posible del cultivo (lo cual es difícil de regular dada su dependencia a temperaturas de $18^{\circ} \mathrm{C}$ a $5 \mathrm{~cm}$ de profundidad de suelo) y el atraso de la fecha de siembra de las praderas (mayo) para otorgar mayor tiempo de descomposición de los residuos. Sin embargo, las temperaturas para la germinación de las especies sembradas pueden no ser beneficiosas para su posterior desarrollo, por lo que se dependerá del año para lograr o no establecimientos aceptables.

Si la probabilidad es que el año va a ser lluvioso, con precipitaciones que exceden a las medias, tener presente que se puede afectar notoriamente la población de plantas del género Lotus. En este caso, la utilización de especies como Trifolium repens sería más adecuado.

Con respecto a los factores químicos del suelo, el pH fue el que afectó el establecimiento de las leguminosas. Esto se debe tener en cuenta si se va a realizar una pradera mezcla de gramíneas y leguminosas en chacras con muchos años de secuencia de cultivos agrícolas, donde las fertilizaciones nitrogenadas recurrentes llevan a una paulatina disminución de $\mathrm{pH}$ en los horizontes superficiales.

\section{REFERENCIAS}

Améndola, L., Armentano, S., 2003. Implantación y producción de especies forrajeras sobre rastrojos de cultivos de verano en sistema de siembra directa. Tesis Ing. Agr., Universidad de la República (Uruguay). 83p.

Askin, D., 1994. Pasture Establishment, in: Langer, R.H.M. (Ed.), Pastures their ecology and management. University Press, Auckland, Oxford, pp. 132-156.

Borges, R., 2001. Descomposición de rastrojos de trigo, soja y maíz sobre suelo en secuencia de cultivos sembrados 
sin laboreo con y sin rotación de pasturas. Tesis Ing. Agr., Universidad de la República (Uruguay). 65p.

Boschi, F., Latorre, P., Saldanha, S., Machado, J., Bentancur, O., Moure, S., 2016. Importancia de las semillas duras en leguminosas forrajeras producidas en Uruguay. Agrociencia Uruguay 20(2), 43-50.

Bray, R., Kurtz, L., 1945. Determination of total, organic, and available forms of phosphorus in soils. Soil Science 59(1), 39-46.

Brock, J., Anderson, L., Lancashire, J., 1982. Grasslands Roa' tall fescue: Seedling growth and establishment. New Zealand Journal of Experimental Agriculture 3(10), 285-289.

Carámbula, M., 1991. Aspectos relevantes para producción forrajera. Serie Técnica $N^{\circ} 19$, Instituto Nacional de Investigación Agropecuaria (INIA), Montevideo, Uruguay, pp. 34-40. http://www.ainfo.inia.uy/digital/bitstream/ item/2921/1/111219220807114541.pdf

Comisión Nacional de Estudio Agronómico de la Tierra (CONEAT), 1979. Grupos de suelos CONEAT. Índices de productividad. Comisión Nacional de Estudio Agronómico de la Tierra, Ministerios de Agricultura y Pesca (CONEAT-MAP), Montevideo, Uruguay.

Deregibus, V., Casal, J., Jacobo, E., Gibson, D., Kauffman, M., Rodríguez, A., 1994. Evidence that Heavy Grazing May Promote the Germination of Lolium multiflorum Seeds via Phytochrome-Mediated Perception of High Red/FarRed Ratios. Functional Ecology 8(4), 536-542.

De Wit, C.T., 1960. On competition. Verslagen landbouwkundig Onderzoek. 66(8), 1-82.

Donald, C., 1963. Competition Among Crop and Pasture plants. Advances in Agronomy 15, 1-118.

Everitt, B., Hothorn, T., 2011a. Cluster Analysis, in: Everitt, B., Hothorn, T. (Eds.), An introduction to Applied Multivariate Analysis with R. Springer, New York, pp. 162-200.

Everitt, B., Hothorn, T., 2011b. Principal Components Analysis, in: Everitt, B., Hothorn, T. (Eds.), An introduction to Applied Multivariate Analysis with R. Springer, New York, pp. 61-102.

Flores, P.G., López, I.F., Kemp, P.D., Dörner, J., Zhang, B., 2016. Modelo de Árbol de Decisión: una herramienta para el manejo de la pradera. Agro Sur 44(2), 3-10.

Formoso, F., 2010. Festuca arundinacea, manejo para producción de forraje y semillas. Serie Técnica $N^{\circ} 182$, INIA, Montevideo, Uruguay, pp. 44-51. http://www. inia.uy/Publicaciones/Documentos \%20 compartidos/18429081210150440.pdf

Formoso, F., 2007. Jornada instalación y manejo de pasturas. Serie actividades de difusión $N^{\circ} 483$, INIA La Estanzuela, Colonia, Uruguay, pp. 19-39. http://www.ainfo.inia.uy/digital/bitstream/item/494/1/111219220807120047.pdf

Garnier, E., 1992. Growth analysis of congeneric annual and perennial grass species. Journal of Ecology 80(4), 665-675.

George, E., Horst, W., Neumann, E., 2012. Adaptation of Plants to Adverse Chemical Soil Conditions, in: Marschner, $\mathrm{H}$. (Ed.), Marschner's Mineral Nutrition of Higher Plants: $3^{\text {rd }}$ Edition, Elsevier, Academic Press, Hohenheim, Ger- many, pp. 409-472.

Harper, J., Benton, R., 1966. The behaviour of seeds in soil. II. The germination of seeds on the surface of a water supplying substrate. Journal of Ecology 54(1), 151-166.

Harris, W., 1994. Pasture as an ecosystem, in: Langer, R.H.M. (Ed.), Pastures their ecology and management. University Press, Auckland, Oxford, pp. 75-131.

Loydi, A., Eckstein, R.L., Otte, A., Donath, T.W., 2013. Effects of litter on seedling establishment in natural and seminatural grasslands: A meta-analysis. Journal of Ecology 101(2), 454-464.

Miglécz, T., Tóthmérész, B., Valkó, O., Kelemen, A., Török, P., 2013. Effects of litter on seedling establishment: An indoor experiment with short-lived Brassicaceae species. Plant Ecology 214, 189-193.

Milchunas, D.G., Vandever, M.W., Ball, L.O., Hyberg, S., 2011. Allelopathic Cover Crop Prior to Seeding Is More Important Than Subsequent Grazing/Mowing in Grassland Establishment. Rangeland Ecology \& Management 64(3), 291-300.

Ministerio de Ganadería, Agricultura y Pesca, Dirección de Investigaciones Económicas Agropecuarias) (MGAP/ DIEA), 2015. Anuario Estadístico Agropecuario 2015. Montevideo, Uruguay.

Morón, A., 2001. El rol de los rastrojos en la fertilidad del suelo, in: Díaz, R. (Ed.), Siembra directa en el Cono Sur. PROCISUR, Montevideo, Uruguay, pp. 387-406.

Parr, J.F., Papendik, R.I., 1978. Factors affecting the decomposition of crop residues by microorganisms, in: Oschwald, W.R. (Ed.), Crop Residue Management Systems. ASA Special Publication Number 31, Madison, Wisconsin, pp. 101-129.

Patrick, Z., 1971. Phytotoxic Substances Associated with the Decomposition in Soil of Plant Residues. Soil Science 111(1), 13-18.

Pérez, C., De La Fuente, L., Arias, A., Altier, N., 2001. Uso de Pseudomonas fluorescentes nativas para el control de enfermedades de implantación en Lotus corniculatus L. Agrociencia Uruguay 5(1), 41-47.

Pozzolo, 0., 2006. Recomendaciones para implantación de pasturas. INTA E.E.A Concepción del Uruguay. Hoja Informativa Electrónica, 6(143), 1-4. http://www.produccion-animal.com.ar/produccion_y_manejo_pasturas / pasturas\%20artificiales/42-implantacion_praderas.pdf

Satchell, J., 1974. Introduction: litter--interface of animate/ inanimate matter. Biology of Plant Litter Decomposition 1974(1), 13-44.

Walkley, A., Black, I., 1934. An examination of the Degtjareff method for determining soil organic matter, and a proposed modification of the chromic acid titration method. Soil Science 37(1), 29-38.

Wright, R.J., 1989. Soil aluminum toxicity and plant growth. Communications in Soil Science and Plant Analysis 20(15-16), 1479-1497.

Zanoniani, R., 2010. Estado actual del conocimiento en producción de pasturas, brecha tecnológica. Agrociencia Uruguay 14(3), 26-30. 